Article

\title{
Existence Results for Sequential Riemann-Liouville and Caputo Fractional Differential Inclusions with Generalized Fractional Integral Conditions
}

\author{
Jessada Tariboon ${ }^{1, *,+}\left(\mathbb{D}\right.$, Sotiris K. Ntouyas ${ }^{2,3,+}+\mathbb{C}$, Bashir Ahmad ${ }^{3,+}\left(\mathbb{D}\right.$ and Ahmed Alsaedi ${ }^{3,+}+\mathbb{C}$ \\ 1 Intelligent and Nonlinear Dynamic Innovations Research Center, Department of Mathematics, Faculty of \\ Applied Science, King Mongkut's University of Technology North Bangkok, Bangkok 10800, Thailand \\ 2 Department of Mathematics, University of Ioannina, 45110 Ioannina, Greece; sntouyas@uoi.gr \\ 3 Nonlinear Analysis and Applied Mathematics (NAAM)-Research Group, Department of Mathematics, \\ Faculty of Science, King Abdulaziz University, P.O. Box 80203, Jeddah 21589, Saudi Arabia; \\ bashirahmad_qau@yahoo.com (B.A.); aalsaedi@hotmail.com (A.A.) \\ * Correspondence: jessada.t@sci.kmutnb.ac.th \\ + These authors contributed equally to this work.
}

Received: 7 May 2020; Accepted: 22 June 2020; Published: 26 June 2020

\begin{abstract}
Under different criteria, we prove the existence of solutions for sequential fractional differential inclusions containing Riemann-Liouville and Caputo type derivatives and supplemented with generalized fractional integral boundary conditions. Our existence results rely on the endpoint theory, the Krasnosel'skii's fixed point theorem for multivalued maps and Wegrzyk's fixed point theorem for generalized contractions. We demonstrate the application of the obtained results with the help of examples.
\end{abstract}

Keywords: Riemann-Liouville fractional derivative; Caputo fractional derivative; inclusions; endpoint theory; generalized fractional integral; Krasnosel'skií's multi-valued fixed point theorem; Wegrzyk's fixed point theorem

\section{Introduction}

Boundary value problems involving fractional differential equations and inclusions with a variety of boundary conditions have been investigated in recent years. For application details, we refer the reader to [1-7] and the references cited therein, while some recent results on boundary value problems of fractional differential inclusions can be found in [8-21].

In a recent work [22], the authors studied the existence of solutions for a nonlinear sequential Riemann-Liouville and Caputo fractional differential equation subject to generalized fractional integral conditions. The objective of the present paper is to investigate the multivalued analogue of the problem considered in [22]. Precisely, we consider the following inclusions problem:

$$
\begin{aligned}
& { }^{R L} D^{q}\left({ }^{C} D^{r} x\right)(t) \in F(t, x(t)), \quad 0<q \leq 1,0<r \leq 1, t \in(0, T), \\
& x(0)=\sum_{i=1}^{m} \gamma_{i} \bar{\rho}_{i} I_{\overline{\eta_{i}}, \bar{\kappa}_{i}}^{\bar{\beta}_{i}, \bar{\beta}_{i}} x\left(\xi_{i}\right), \quad x(T)=\sum_{j=1}^{n} \sigma_{j}{ }^{\rho_{j}} I_{\eta_{j}, \kappa_{j}}^{\alpha_{j}, \beta_{j}} x\left(\delta_{j}\right),
\end{aligned}
$$

where ${ }^{R L} D^{q}$ and ${ }^{C} D^{r}$ denote the left Riemann-Liouville and left Caputo fractional derivatives of order $q$ and $r$, respectively; $F:[0, T] \times \mathbb{R} \rightarrow \mathcal{P}(\mathbb{R})$ is a multivalued map; $\mathcal{P}(\mathbb{R})$ is the family of all nonempty subsets of $\mathbb{R} ; \tilde{\rho} I_{\tilde{\eta}, \tilde{\kappa}}^{\tilde{\alpha}, \tilde{\beta}}$ is the generalized fractional integral of order $\tilde{\alpha}>0$ with parameters $\tilde{\rho} \in\left\{\bar{\rho}_{i}, \rho_{j}\right\}, \tilde{\alpha} \in\left\{\bar{\alpha}_{i}, \alpha_{j}\right\}>0, \tilde{\beta} \in\left\{\bar{\beta}_{i}, \beta_{j}\right\}, \tilde{\eta} \in\left\{\bar{\eta}_{i}, \eta_{j}\right\}, \tilde{\kappa} \in\left\{\bar{\kappa}_{i}, \kappa_{j}\right\} \in \mathbb{R}, \gamma_{i}, \sigma_{j} \in \mathbb{R}$ and $\tilde{\xi}_{i}, \delta_{j} \in(0, T)$, 
for all $i=1,2, \ldots, m, j=1,2, \ldots, n$. For definitions of fractional derivatives and integrals involved in the problem (1) and (2), see [22]. Here we emphasize that the boundary conditions (2) correspond to different kinds of integral boundary conditions for appropriate choice of the parameters; for details, see Remark 2 in [22].

In the sequel, we need the following known lemma.

Lemma 1. [22] Let $0<q \leq 1,0<r \leq 1, \bar{\rho}_{i}, \rho_{j}, q, r, \bar{\alpha}_{i}, \alpha_{j}>0, \xi_{i}, \delta_{j} \in(0, T), \bar{\beta}_{i}, \bar{\eta}_{i}, \bar{\kappa}_{i}, \beta_{j}, \eta_{j}, \kappa_{j} \in \mathbb{R}$ for $i=1,2, \ldots, m, j=1,2, \ldots, n$, and $y \in C([0, T], \mathbb{R})$. The unique solution of the following linear sequential fractional differential equation

$$
{ }^{R L} D^{q}\left({ }^{C} D^{r} x\right)(t)=y(t), \quad t \in(0, T),
$$

subject to the generalized fractional integral conditions

$$
x(0)=\sum_{i=1}^{m} \gamma_{i} \bar{\rho}_{i} I_{\bar{\eta}_{i}, \bar{\kappa}_{i}, \bar{\beta}_{i}} x\left(\xi_{i}\right), \quad x(T)=\sum_{j=1}^{n} \sigma_{j} \rho_{j} I_{\eta_{j}, \kappa_{j}}^{\alpha_{j}, \beta_{j}} x\left(\delta_{j}\right),
$$

is given by the formula

$$
\begin{aligned}
x(t)= & \frac{1}{\Omega}\left[\left(\Omega_{1}-\Omega_{2} \frac{\Gamma(q)}{\Gamma(q+r)} t^{q+r-1}\right){ }^{R L} I^{q+r} y(T)\right. \\
& +\left(\Omega_{2} \frac{\Gamma(q)}{\Gamma(q+r)} t^{q+r-1}-\Omega_{1}\right) \sum_{j=1}^{n} \sigma_{j} \rho_{j} I_{\eta_{j}, \kappa j}^{\alpha_{j}, \beta_{j} R L} I^{q+r} y\left(\delta_{j}\right) \\
& \left.+\left(\Omega_{3}-\Omega_{4} \frac{\Gamma(q)}{\Gamma(q+r)} t^{q+r-1}\right) \sum_{i=1}^{m} \gamma_{i}{ }^{\rho_{i}} I_{\bar{\eta}_{i}, \bar{\beta}_{i}}^{\bar{\alpha}_{i}, \bar{\beta}_{i} R L} I^{q+r} y\left(\xi_{i}\right)\right]+{ }^{R L} I^{q+r} y(t),
\end{aligned}
$$

where it is assumed that $\Omega=\Omega_{1} \Omega_{4}-\Omega_{2} \Omega_{3} \neq 0$,

$$
\begin{aligned}
& \Omega_{1}=\frac{\Gamma(q)}{\Gamma(q+r)} \sum_{i=1}^{m} \gamma_{i} \pi_{q+r-1}^{\bar{\rho}_{i}, \bar{\alpha}_{i}, \bar{\beta}_{i}, \bar{\eta}_{i}, \bar{\kappa}_{i}}\left(\xi_{i}\right), \quad \Omega_{2}=\sum_{i=1}^{m} \gamma_{i} \pi_{0}^{\bar{\rho}_{i}, \bar{\alpha}_{i}, \bar{\beta}_{i}, \bar{\eta}_{i}, \bar{\kappa}_{i}}\left(\xi_{i}\right)-1, \\
& \Omega_{3}=\frac{\Gamma(q)}{\Gamma(q+r)}\left(\sum_{j=1}^{n} \sigma_{j} \pi_{q+r-1}^{\rho_{j}, \alpha_{j}, \beta_{j}, \eta_{j}, \kappa_{j}}\left(\delta_{j}\right)-T^{q+r-1}\right), \\
& \Omega_{4}=\sum_{j=1}^{n} \sigma_{j} \pi_{0}^{\rho_{j}, \alpha_{j}, \beta_{j}, \eta_{j}, \kappa_{j}}\left(\delta_{j}\right)-1, \pi_{m}^{\rho, \alpha, \beta, \eta, \kappa}(t)=\rho^{-\beta} \frac{\Gamma\left(\frac{\rho \eta+\rho+m}{\rho}\right)}{\Gamma\left(\frac{\rho \eta+\rho \alpha+\rho+m}{\rho}\right)} t^{\rho(\alpha+\eta)+m+\kappa} .
\end{aligned}
$$

In view of Lemma 1, we can define a solution of problem (1) and (2) as follows.

Definition 1. A function $x \in C^{1}([0, T], \mathbb{R})$ is called a solution of problem (1) and (2) if there exists a function $v \in L^{1}([0, T], \mathbb{R})$ with $v(t) \in F(t, x(t))$, a.e. in $[0, T]$ such that $x(0)=\sum_{i=1}^{m} \gamma_{i}{ }_{i}^{\bar{\rho}} I_{\bar{\eta}_{i}, \bar{\kappa}_{i}}^{\bar{\alpha}_{i} \bar{\beta}_{i}} x\left(\xi_{i}\right), \quad x(T)=$ $\sum_{j=1}^{n} \sigma_{j} \rho_{j} I_{\eta_{j}, r_{j}}^{\alpha_{j}, \beta_{j}} x\left(\delta_{j}\right)$, and 


$$
\begin{aligned}
x(t)= & \frac{1}{\Omega}\left(\left(\Omega_{1}-\Omega_{2} \frac{\Gamma(q)}{\Gamma(q+r)} t^{q+r-1}\right) R I^{q+r} v(T)\right. \\
& +\left(\Omega_{2} \frac{\Gamma(q)}{\Gamma(q+r)} t^{q+r-1}-\Omega_{1}\right) \sum_{j=1}^{n} \sigma_{j} \rho_{j} I_{\eta_{j}, \kappa_{j}}^{\alpha_{j}, \beta_{j} R L} I^{q+r} v\left(\delta_{j}\right) \\
& \left.+\left(\Omega_{3}-\Omega_{4} \frac{\Gamma(q)}{\Gamma(q+r)} t^{q+r-1}\right) \sum_{i=1}^{m} \gamma_{i}{ }^{\bar{\rho}_{i}} I_{\bar{\eta}_{j}, \bar{\kappa}_{i}}^{\bar{\alpha}_{i}, \bar{\beta}_{i} R L} I^{q+r} v\left(\xi_{i}\right)\right)+{ }^{R L} I^{q+r} v(t) .
\end{aligned}
$$

For the sake of computational convenience, we set

$$
\begin{aligned}
\Phi= & \frac{1}{\Gamma(q+r+1)|\Omega|}\left(\left|\Omega_{1}\right| T^{q+r}+\left|\Omega_{2}\right| \frac{\Gamma(q)}{\Gamma(q+r)} T^{2 q+2 r-1}\right. \\
& +\left[\left|\Omega_{2}\right| \frac{\Gamma(q)}{\Gamma(q+r)} T^{q+r-1}+\left|\Omega_{1}\right|\right] \sum_{j=1}^{n}\left|\sigma_{j}\right| \pi_{q+r}^{\rho_{j}, \alpha_{j}, \beta_{j}, \eta_{j}, \kappa_{j}}\left(\delta_{j}\right) \\
& \left.+\left[\left|\Omega_{3}\right|+\left|\Omega_{4}\right| \frac{\Gamma(q)}{\Gamma(q+r)} T^{q+r-1}\right] \sum_{i=1}^{m}\left|\gamma_{i}\right| \pi_{q+r}^{\bar{\rho}_{i}, \bar{\alpha}_{i}, \bar{\beta}_{i}, \bar{\eta}_{i}, \bar{\kappa}_{i}}\left(\xi_{i}\right)\right)+\frac{T^{q+r}}{\Gamma(q+r+1)}
\end{aligned}
$$

\section{Main Results}

In this section, we present our main results in different subsections by applying a variety of fixed point theorems for multivalued maps.

\subsection{Existence Result via Endpoint Theory}

Denote by $\mathcal{Z}:=C([0, T], \mathbb{R})$ the Banach space of all continuous functions from $[0, T]$ into $\mathbb{R}$ with the norm $\|x\|=\sup \{|x(t)|, t \in[0, T]\}$. The space of functions $x:[0, T] \rightarrow \mathbb{R}$ such that $\|x\|_{L^{1}}=\int_{0}^{T}|x(t)| d t$ is denoted by $L^{1}([0, T], \mathbb{R})$.

Let $(X, d)$ be a metric space induced from the normed space $(X ;\|\cdot\|)$. Consider $H_{d}: \mathcal{P}(X) \times$ $\mathcal{P}(X) \rightarrow \mathbb{R} \cup\{\infty\}$ given by $H_{d}(A, B)=\max \left\{\sup _{a \in A} d(a, B), \sup _{b \in B} d(A, b)\right\}$, where $d(A, b)=$ $\inf _{a \in A} d(a ; b)$ and $d(a, B)=\inf _{b \in B} d(a ; b)$. Then $\left(\mathcal{P}_{c l, b}(X), H_{d}\right)$ is a metric space (see [23]).

Now we state the endpoint fixed point theorem that will be applied to prove our first result.

Lemma 2. ([24]) Let $(X, d)$ be a complete metric space and $\mathcal{S}: X \rightarrow \mathcal{P}_{c l, b d}(X)$ be a multifunction such that $H_{d}(\mathcal{S} x, \mathcal{S} y) \leq \psi(d(x, y))$ for all $x, y \in X$, where $\mathcal{P}_{c l, b d}(X)$ is the collection of all nonempty closed and bounded subsets of $X$ and $\psi:[0, \infty) \rightarrow[0, \infty)$ is an upper semi-continuous function such that $\psi(t)<t$ and $\liminf _{t \rightarrow \infty}(t-\psi(t))>0$ for all $t>0$. Then $\mathcal{S}$ has a unique endpoint if and only if $\mathcal{S}$ has an approximate endpoint property.

For more details about endpoint theory, we refer the reader to the article [25].

By Lemma 1, we define an operator $\mathcal{G}: \mathcal{Z} \rightarrow \mathcal{P}(\mathcal{Z})$ as follows:

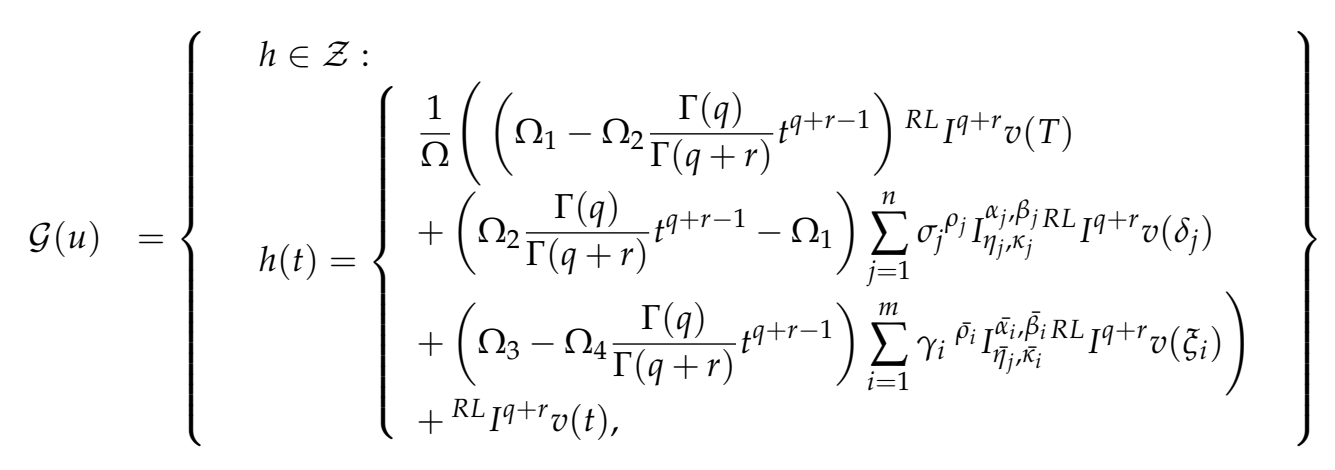


for $v \in S_{F, u}$, where $S_{F, u}$ denotes the set of selections of $F$ defined by

$$
S_{F, u}:=\left\{v \in L^{1}([0, T], \mathbb{R}): v(t) \in F(t, u(t)) \text { for a.e. } t \in[0, T]\right\} .
$$

Theorem 1. Suppose that $\psi:[0, \infty) \rightarrow[0, \infty)$ is a nondecreasing upper semi-continuous mapping such that $\liminf _{t \rightarrow \infty}(t-\psi(t))>0$ and $\psi(t)<t$ for all $t>0$. Moreover, assume that $F:[0, T] \times \mathbb{R} \rightarrow \mathcal{P}_{c p}(\mathbb{R})$ is an integrable bounded multifunction such that $F(\cdot, u):[0, T] \rightarrow \mathcal{P}_{c p}(\mathbb{R})$ is measurable for all $u \in \mathbb{R} ;$ here $\mathcal{P}_{c p}(\mathbb{R})$ denotes the collection of all nonempty compact subsets of $\mathbb{R}$. In addition, we assume that there exists a function $\ell \in C([0, T],[0, \infty))$ such that

$$
H_{d}(F(t, u(t))-F(t, v(t))) \leq(\|\ell\| \Phi)^{-1} \ell(t) \psi(|u(t)-v(t)|),
$$

where $\Phi$ is defined by (6). If the multifunction $\mathcal{G}$ (defined by (7)) has the approximate endpoint property, then the inclusion problem (1) and (2) has a solution.

Proof. Our proof will be complete once it is shown that the multifunction $\mathcal{G}: \mathcal{Z} \rightarrow \mathcal{P}(\mathcal{Z})$ defined by (7) has an endpoint. To do this, we show that the operator $\mathcal{G}(u)$ is a closed subset of $\mathcal{P}(\mathcal{Z})$ for all $u \in \mathcal{Z}$. Note that the multivalued map $t \mapsto F(t, u(t))$ is measurable and has closed values for all $u \in \mathcal{Z}$, and therefore has a measurable selection. So $S_{F, u}$ is nonempty for all $u \in \mathcal{Z}$. Let $\left\{z_{n}\right\}_{n \geq 1}$ be a sequence in $\mathcal{G}(u)$ with $z_{n} \rightarrow z$ for $u \in \mathcal{Z}$. For every $n \in \mathbb{N}$, choose $v_{n} \in S_{F, u_{n}}$ such that

$$
\begin{aligned}
z_{n}(t)= & \frac{1}{\Omega}\left(\left(\Omega_{1}-\Omega_{2} \frac{\Gamma(q)}{\Gamma(q+r)} t^{q+r-1}\right) R L I^{q+r} v_{n}(T)\right. \\
& +\left(\Omega_{2} \frac{\Gamma(q)}{\Gamma(q+r)} t^{q+r-1}-\Omega_{1}\right) \sum_{j=1}^{n} \sigma_{j} \rho_{j} I_{\eta_{j}, \kappa_{j}}^{\alpha_{j}, \beta_{j} R L} I^{q+r} v_{n}\left(\delta_{j}\right) \\
& \left.+\left(\Omega_{3}-\Omega_{4} \frac{\Gamma(q)}{\Gamma(q+r)} t^{q+r-1}\right) \sum_{i=1}^{m} \gamma_{i}{ }^{\overline{\rho_{i}}} I_{\bar{\eta}_{j}, \bar{\kappa}_{i}}^{\bar{\alpha}_{i}, \bar{\beta}_{i} R L} I^{q+r} v_{n}\left(\xi_{i}\right)\right)+{ }^{R L} I^{q+r} v_{n}(t) .
\end{aligned}
$$

In view of the compactness of $F$, we deduce that the sequence $\left\{v_{n}\right\}_{n \geq 1}$ has a subsequence which converges to some $v \in L^{1}([0, T])$. Let this subsequence be denoted by $\left\{v_{n}\right\}_{n \geq 1}$ again. Clearly $v \in S_{F, u}$ and for all $t \in[0, T]$,

$$
\begin{aligned}
z_{n}(t) \rightarrow z(t)= & \frac{1}{\Omega}\left(\left(\Omega_{1}-\Omega_{2} \frac{\Gamma(q)}{\Gamma(q+r)} t^{q+r-1}\right) R{ }^{R L} I^{q+r} v(T)\right. \\
& +\left(\Omega_{2} \frac{\Gamma(q)}{\Gamma(q+r)} t^{q+r-1}-\Omega_{1}\right) \sum_{j=1}^{n} \sigma_{j} \sigma_{j}^{\rho_{j}} I_{\eta_{j}, \kappa_{j}}^{\alpha_{j}, \beta_{j} R L} I^{q+r} v\left(\delta_{j}\right) \\
& \left.+\left(\Omega_{3}-\Omega_{4} \frac{\Gamma(q)}{\Gamma(q+r)} t^{q+r-1}\right) \sum_{i=1}^{m} \gamma_{i} \bar{\rho}^{\bar{\rho}_{i}} I_{\bar{\eta}_{j}, \bar{\kappa}_{i}, \bar{\kappa}_{i} R L} I^{q+r} v\left(\xi_{i}\right)\right)+{ }^{R L} I^{q+r} v(t) .
\end{aligned}
$$

Thus $z \in \mathcal{G}(u)$ and consequently $\mathcal{G}$ is closed-valued. On the other hand, $\mathcal{G}(u)$ is a bounded set for all $u \in \mathcal{Z}$ as $F$ is a compact multivalued map.

Now we establish that $H_{d}(\mathcal{G}(u), \mathcal{G}(w)) \leq \psi(\|u-w\|)$. Let $u, \omega \in \mathcal{Z}$ and $h_{1} \in \mathcal{G}(\omega)$. Choose $v_{1} \in S_{F, \omega}$ such that 


$$
\begin{aligned}
z_{1}(t)= & \frac{1}{\Omega}\left(\left(\Omega_{1}-\Omega_{2} \frac{\Gamma(q)}{\Gamma(q+r)} t^{q+r-1}\right) R L I^{q+r} v_{1}(T)\right. \\
& +\left(\Omega_{2} \frac{\Gamma(q)}{\Gamma(q+r)} t^{q+r-1}-\Omega_{1}\right) \sum_{j=1}^{n} \sigma_{j} \rho_{j} I_{\eta_{j}, \kappa_{j}}^{\alpha_{j}, \beta_{j} R L} I^{q+r} v_{1}\left(\delta_{j}\right) \\
& \left.+\left(\Omega_{3}-\Omega_{4} \frac{\Gamma(q)}{\Gamma(q+r)} t^{q+r-1}\right) \sum_{i=1}^{m} \gamma_{i}{ }^{\bar{\rho}_{i}} I_{\eta_{j}, \kappa_{i}}^{\bar{\alpha}_{i}, \bar{\beta}_{i} R L} I^{q+r} v_{1}\left(\xi_{i}\right)\right)+{ }^{R L} I^{q+r} v_{1}(t)
\end{aligned}
$$

for almost all $t \in[0, T]$. Since

$$
H_{d}(F(t, u(t))-F(t, \omega(t))) \leq(\|\ell\| \Phi)^{-1} \ell(t) \psi(|u(t)-\omega(t)|), \text { for all } t \in[0, T],
$$

there exists $z \in F(t, u(t))$ provided that

$$
\left|v_{1}(t)-z\right| \leq(\|\ell\| \Phi)^{-1} \ell(t) \psi(|u(t)-\omega(t)|), \text { for all } t \in[0, T] .
$$

Let us consider the multivalued map $\mathcal{V}:[0, T] \rightarrow \mathcal{P}(\mathbb{R})$ given by

$$
\mathcal{V}(t)=\left\{z \in \mathbb{R}:\left|v_{1}(t)-z\right| \leq(\|\ell\| \Phi)^{-1} \ell(t) \psi(|u(t)-\omega(t)|)\right\} .
$$

The multifunction $\mathcal{V}(\cdot) \cap F(\cdot, u(\cdot))$ is measurable, since $v_{1}$ and $\varphi=(\|\ell\| \Phi)^{-1} \ell \psi(|u-\omega|)$ are measurable. Choose $v_{2}(t) \in F(t, u(t))$ such that

$$
\left|v_{1}(t)-v_{2}(t)\right| \leq(\|\ell\| \Phi)^{-1} \ell(t) \psi(|u(t)-\omega(t)|), \text { for all } t \in[0, T] .
$$

We define the element $h_{2} \in \mathcal{G}(u)$ as follows:

$$
\begin{aligned}
z_{2}(t)= & \frac{1}{\Omega}\left(\left(\Omega_{1}-\Omega_{2} \frac{\Gamma(q)}{\Gamma(q+r)} t^{q+r-1}\right) R L I^{q+r} v_{2}(T)\right. \\
& +\left(\Omega_{2} \frac{\Gamma(q)}{\Gamma(q+r)} t^{q+r-1}-\Omega_{1}\right) \sum_{j=1}^{n} \sigma_{j} \rho_{j} I_{\eta_{j}, r_{j}}^{\alpha_{j}, \beta_{j} R L} I^{q+r} v_{2}\left(\delta_{j}\right) \\
& \left.+\left(\Omega_{3}-\Omega_{4} \frac{\Gamma(q)}{\Gamma(q+r)} t^{q+r-1}\right) \sum_{i=1}^{m} \gamma_{i}{ }^{\overline{\rho_{i}}} I_{\bar{\eta}_{j}, \bar{\kappa}_{i}}^{\bar{\alpha}_{i}, \bar{\beta}_{i} R L} I^{q+r} v_{2}\left(\xi_{i}\right)\right) \\
& +{ }^{R L} I^{q+r} v_{2}(t), \quad \text { for all } \quad t \in[0, T] .
\end{aligned}
$$

Let $\sup _{t \in[0, T]}|\ell(t)|=\|\ell\|$. Then, one can get 


$$
\begin{aligned}
& \left|z_{1}(t)-z_{2}(t)\right| \\
& \leq \frac{1}{|\Omega|}\left(\left[\left|\Omega_{1}\right|+\left|\Omega_{2}\right| \frac{\Gamma(q)}{\Gamma(q+r)} T^{q+r-1}\right] R L I^{q+r}\left(\left|v_{1}-v_{2}\right|\right)(T)\right. \\
& +\left[\left|\Omega_{2}\right| \frac{\Gamma(q)}{\Gamma(q+r)} T^{q+r-1}+\left|\Omega_{1}\right|\right] \sum_{j=1}^{n}\left|\sigma_{j}\right| \rho_{j}^{\rho_{j}} I_{\eta_{j}, \beta_{j}}^{\alpha_{j}, \beta_{j}} R L I^{q+r}\left(\left|v_{1}-v_{2}\right|\right)\left(\delta_{j}\right) \\
& \left.+\left[\left|\Omega_{3}\right|+\left|\Omega_{4}\right| \frac{\Gamma(q)}{\Gamma(q+r)} T^{q+r-1}\right] \sum_{i=1}^{m}\left|\gamma_{i}\right|^{\bar{\rho}_{i}} I_{\bar{\eta}_{i}, \bar{\kappa}_{i}}^{\bar{\alpha}_{i} \bar{\beta}_{i} R L} I^{q+r}\left(\left|v_{1}-v_{2}\right|\right)\left(\xi_{i}\right)\right) \\
& +{ }^{R L} I^{q+r}\left(\left|v_{1}-v_{2}\right|\right)(t) \\
& \leq\|\ell\|\left\|v_{1}-v_{2}\right\|\left\{\frac { 1 } { \Gamma ( q + r + 1 ) | \Omega | } \left(\left|\Omega_{1}\right| T^{q+r}+\left|\Omega_{2}\right| \frac{\Gamma(q)}{\Gamma(q+r)} T^{2 q+2 r-1}\right.\right. \\
& +\left[\left|\Omega_{2}\right| \frac{\Gamma(q)}{\Gamma(q+r)} T^{q+r-1}+\left|\Omega_{1}\right|\right] \times \\
& \times \sum_{j=1}^{n}\left|\sigma_{j}\right| \rho_{j}^{\left(-\beta_{j}\right)} \delta_{j}^{\rho_{j}\left(\alpha_{j}+\eta_{j}\right)+\kappa_{j}+q+r} \frac{\Gamma\left(\frac{\rho_{j} \eta_{j}+\rho_{j}+q+r}{\rho_{j}}\right)}{\Gamma\left(\frac{\rho_{j} \eta_{j}+\rho_{j} \alpha_{j}+\rho_{j}+q+r}{\rho_{j}}\right)} \\
& +\left[\left|\Omega_{3}\right|+\left|\Omega_{4}\right| \frac{\Gamma(q)}{\Gamma(q+r)} T^{q+r-1}\right] \times \\
& \left.\left.\times \sum_{i=1}^{m}\left|\gamma_{i}\right| \mid \bar{\rho}_{i}\left(-\bar{\beta}_{i}\right) \xi_{i}^{\bar{\rho}_{i}\left(\bar{\alpha}_{i}+\bar{\eta}_{i}\right)+\bar{\kappa}_{i}+q+r} \frac{\Gamma\left(\frac{\bar{\rho}_{i} \bar{\eta}_{i}+\bar{\rho}_{i}+q+r}{\bar{\rho}_{i}}\right)}{\Gamma\left(\frac{\bar{\rho}_{i} \bar{\eta}_{i}+\bar{\rho}_{i} \bar{c}_{i}+\bar{\rho}_{i}+q+r}{\bar{\rho}_{i}}\right)}\right)+\frac{T^{q+r}}{\Gamma(q+r+1)}\right\} \\
& \leq(\|\ell\| \Phi)^{-1} \|(\ell \| \Phi) \psi(\|u-\omega\|) \\
& =\psi(\|u-\omega\|) \text {, }
\end{aligned}
$$

which implies that $\left\|z_{1}-z_{2}\right\| \leq \psi(\|u-\omega\|)$. Therefore $H_{d}(\mathcal{G}(u), \mathcal{G}(\omega)) \leq \psi(\|u-\omega\|)$ for all $u, \omega \in \mathcal{Z}$. Therefore there exists $u^{*} \in \mathcal{Z}$ such that $\mathcal{G}\left(u^{*}\right)=\left\{u^{*}\right\}$, since the multifunction $\mathcal{G}$ (by the hypothesis) has an approximate endpoint property. Therefore we deduce that the problem (1) and (2) has a solution $u^{*}$. The proof is complete.

Example 1. Consider the following inclusions problem containing Riemann-Liouville and Caputo derivative operators with generalized fractional integral boundary conditions

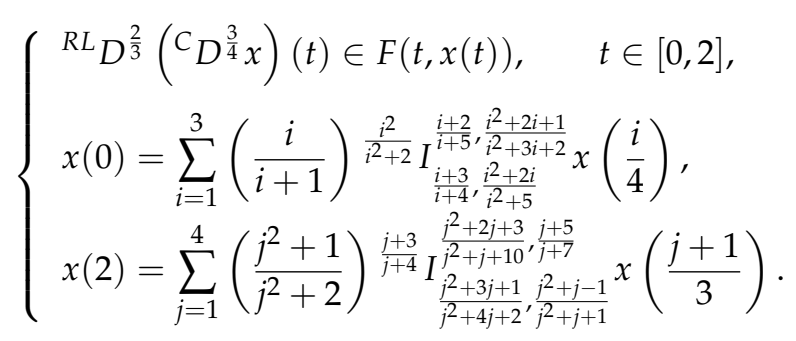

Here $q=2 / 3, r=3 / 4, T=2, m=3, n=4, \gamma_{i}=(i /(i+1)), \bar{\rho}_{i}=\left(i^{2} /\left(i^{2}+2\right)\right), \bar{\alpha}_{i}=((i+2) /(i+$ $5)), \bar{\beta}_{i}=\left(\left(i^{2}+2 i+1\right) /\left(i^{2}+3 i+2\right)\right), \bar{\eta}_{i}=((i+3) /(i+4)), \bar{\kappa}_{i}=\left(\left(i^{2}+2 i\right) /\left(i^{2}+5\right)\right), \xi_{i}=(i / 4)$, $i=1, \ldots, 3, \sigma_{j}=\left(\left(j^{2}+1\right) /\left(j^{2}+2\right)\right), \rho_{j}=((j+3) /(j+4)), \alpha_{j}=\left(\left(j^{2}+2 j+3\right) /\left(j^{2}+j+10\right)\right)$, $\beta_{j}=((j+5) /(j+7)), \eta_{j}=\left(\left(j^{2}+3 j+1\right) /\left(j^{2}+4 j+2\right)\right), \kappa_{j}=\left(\left(j^{2}+j-1\right) /\left(j^{2}+j+1\right)\right), \delta_{j}=$ $((j+1) / 3), j=1, \ldots, 4$. From the given data, we find that $\Omega_{1} \approx 0.7080014810, \Omega_{2} \approx-0.2503200083$, $\Omega_{3} \approx 4.305948722, \Omega_{4} \approx 3.462288181, \Omega \approx 3.529170280$ and $\Phi \approx 4.519175132$.

Let $F:[0,2] \times \mathbb{R} \rightarrow \mathcal{P}(\mathbb{R})$ be a multi-valued map defined by

$$
x \rightarrow F(t, x)=\left[0, \frac{t}{12}\left(\frac{x^{2}+2|x|}{1+|x|}\right)+\frac{1}{2}\right] .
$$


Let us take $\psi(y)=y / 3$ and note that it is nondecreasing upper semi-continuous on $[0,2]$ satisfying $\liminf _{y \rightarrow \infty}(y-\psi(y))>0$ and $\psi(y)<y$ for all $y>0$. Set $\ell(t)=t / 6, t \in[0,2]$ such that $\|\ell\|=1 / 3$. Therefore, we have

$$
H_{d}(F(t, x)-F(t, \bar{x})) \leq \frac{t}{6}|x-\bar{x}|<(\|\ell\| \Phi)^{-1} \frac{t}{6} \psi(|x-\bar{x}|)
$$

for $x, \bar{x} \in \mathbb{R}$. Letting $\mathcal{Z}=C([0,2], \mathbb{R})$, define an operator $\mathcal{G}: \mathcal{Z} \rightarrow \mathcal{P}(\mathcal{Z})$ by

$$
\mathcal{G}(u)=\left\{z \in \mathcal{Z}: \text { there exists } v \in S_{F, u} \text { such that } z(t)=w(t) \text { for all } t \in[0,2]\right\},
$$

where

$$
\begin{aligned}
w(t)= & \frac{1}{\Omega}\left(\left(\Omega_{1}-\Omega_{2} \frac{\Gamma(q)}{\Gamma(q+r)} t^{q+r-1}\right){ }^{R L} I^{q+r} v(T)\right. \\
& +\left(\Omega_{2} \frac{\Gamma(q)}{\Gamma(q+r)} t^{q+r-1}-\Omega_{1}\right) \sum_{j=1}^{n} \sigma_{j} \rho_{j} I_{\eta_{j}, \kappa_{j}}^{\alpha_{j}, \beta_{j} R L} I^{q+r} v\left(\delta_{j}\right) \\
& \left.+\left(\Omega_{3}-\Omega_{4} \frac{\Gamma(q)}{\Gamma(q+r)} t^{q+r-1}\right) \sum_{i=1}^{m} \gamma_{i} \bar{\rho}^{\bar{\rho}_{i}} I_{\bar{\eta}_{j}, \bar{\kappa}_{i}}^{\bar{\alpha}_{i}, \bar{\beta}_{i} R L} I^{q+r} v\left(\xi_{i}\right)\right)+{ }^{R L} I^{q+r} v(t) .
\end{aligned}
$$

Observe that $\inf _{u \in \mathcal{Z}} \sup _{s \in \mathcal{G}(u)}\|u-s\|=0$ in view of $\sup _{u \in \mathcal{G}(0)}\|u\|=0$. Therefore, the operator $\mathcal{G}$ has the approximate endpoint property. Clearly the hypothesis of Theorem 1 is satisfied. So the conclusion of Theorem 1 applies to the problem (8) with $F(t, x)$ given by (9).

\subsection{Existence Result via Krasnosel'skiï's Multi-Valued Fixed Point Theorem}

This subsection is concerned with the second existence result for the problem (1) and (2) when the map $F$ in (1) is $L^{1}$-Carathéodory and convex valued. The proof of this result relies on Krasnosel'skii's fixed point theorem for multivalued maps [26].

In the following we need the following assumptions:

Hypothesis $1(\mathrm{H} 1)$. F : $[0, T] \times \mathbb{R} \rightarrow \mathcal{P}_{c p, c}(\mathbb{R})$ is $L^{1}$-Carathéodory multivalued map, where $\mathcal{P}_{c p, c}(\mathbb{R})$ is the collection of all nonempty compact and convex subsets of $\mathbb{R}$;

Hypothesis $2(\mathrm{H} 2)$. There exists a function $p \in C\left([0, T], \mathbb{R}^{+}\right)$such that

$$
\|F(t, x)\|_{\mathcal{P}}:=\sup \{|w|: w \in F(t, x)\} \leq p(t), \text { for each }(t, x) \in[0, T] \times \mathbb{R}
$$

Hypothesis 3 (H3). There exists a function $k \in \mathcal{Z}$ satisfying $H_{d}(F(t, x), F(t, y)) \leq\|k\|\|x-y\|$, for a.e $t \in[0, T]$ and all $x, y \in \mathcal{Z}$ and that $\|k\|<1 / \Lambda$, where

$$
\begin{aligned}
\Lambda= & \frac{1}{|\Omega|}\left\{\left[\left|\Omega_{2}\right| \frac{\Gamma(q)}{\Gamma(q+r)} T^{q+r-1}+\left|\Omega_{1}\right|\right] \sum_{j=1}^{n}\left|\sigma_{j}\right| \pi_{q+r}^{\rho_{j}, \alpha_{j}, \beta_{j}, \eta_{j}, \kappa_{j}}\left(\delta_{j}\right)\right. \\
& \left.+\left[\left|\Omega_{3}\right|+\left|\Omega_{4}\right| \frac{\Gamma(q)}{\Gamma(q+r)} T^{q+r-1}\right] \sum_{i=1}^{m}\left|\gamma_{i}\right| \pi_{q+r}^{\bar{\rho}_{i}, \bar{\alpha}_{i}, \bar{\beta}_{i}, \bar{\eta}_{i}, \bar{\kappa}_{i}}\left(\xi_{i}\right)+\frac{T^{q+r}}{\Gamma(q+r+1)}\right\} .
\end{aligned}
$$

Theorem 2. If the assumptions H1-H3 are satisfied, then the problem (1) and (2) have at least one solution on $[0, T]$.

Proof. Define the multivalued operators $\mathcal{A}: \mathcal{Z} \longrightarrow \mathcal{P}(\mathcal{Z})$ by 


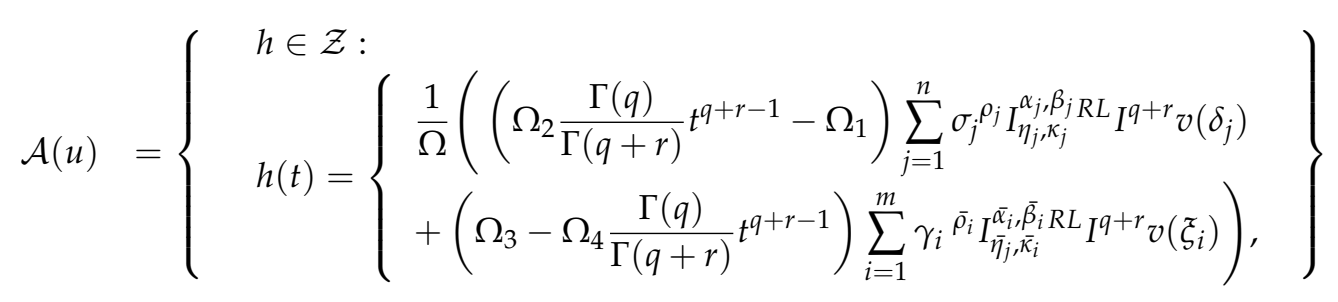

and $\mathcal{B}: \mathcal{Z} \longrightarrow \mathcal{P}(\mathcal{Z})$ by

$$
\mathcal{B}(u)=\left\{\begin{array}{l}
h \in \mathcal{Z}: \\
h(t)=\left\{\begin{array}{l}
\frac{1}{\Omega}\left(\Omega_{1}-\Omega_{2} \frac{\Gamma(q)}{\Gamma(q+r)} t^{q+r-1}\right) R{ }^{R L} I^{q+r} v(T) \\
+{ }^{R L} I^{q+r} v(t)
\end{array}\right\} .
\end{array}\right.
$$

Notice that $\mathcal{G}=\mathcal{A}+\mathcal{B}$, where $\mathcal{G}$ is given by (7). In several steps, it will be shown that $\mathcal{A}$ and $\mathcal{B}$ satisfy the hypothesis of Krasnosel'skii's multivalued fixed point theorem.

Let $B_{r}=\{x \in \mathcal{Z}:\|x\| \leq r\}$ be a bounded set in $\mathcal{Z}$. The operators $\mathcal{A}$ and $\mathcal{B}$ define the multivalued operators $\mathcal{A}, \mathcal{B}: B_{r} \rightarrow \mathcal{P}_{c p, c}(\mathcal{Z})$. Observe that the operator $\mathcal{B}$ is equivalent to the composition $\mathcal{L} \circ S_{F}$, where $\mathcal{L}$ is the continuous linear operator on $L^{1}([0, T], \mathbb{R})$ into $\mathcal{Z}$, defined by

$$
\mathcal{L}(v)(t)=\frac{1}{\Omega}\left(\Omega_{1}-\Omega_{2} \frac{\Gamma(q)}{\Gamma(q+r)} t^{q+r-1}\right) R L I^{q+r} v(T)+{ }^{R L} I^{q+r} v(t) .
$$

For an arbitrary element $x \in B_{r}$, let $\left\{v_{n}\right\}$ be a sequence in $S_{F, x}$. Then $v_{n}(t) \in F(t, x(t))$ for almost all $t \in[0, T]$. As $F(t, x(t))$ is compact for all $t \in J$, we can find a convergent subsequence of $\left\{v_{n}(t)\right\}$ (also labeled as $\left.\left\{v_{n}(t)\right\}\right)$ converging in measure to some $v(t) \in S_{F, x}$ for almost all $t \in[0, T]$. On the other hand, continuity of $\mathcal{L}$ implies that $\mathcal{L}\left(v_{n}\right)(t) \rightarrow \mathcal{L}(v)(t)$ pointwise on $[0, T]$.

To ensure the uniform convergence, we have to establish that $\left\{\mathcal{L}\left(v_{n}\right)\right\}$ is an equi-continuous sequence. Take $\tau_{1}, \tau_{2} \in[0, T]$ with $\tau_{1}<\tau_{2}$ and $x \in B_{r}$. Then

$$
\begin{aligned}
& \left|(\mathcal{L} x)\left(\tau_{2}\right)-(\mathcal{L} x)\left(\tau_{1}\right)\right| \\
\leq & \frac{1}{|\Omega|}\left(\left|\Omega_{2}\right| \frac{\Gamma(q)}{\Gamma(q+r)}\left|\tau_{1}{ }^{q+r-1}-\tau_{2}{ }^{q+r-1}\right|\right){ }^{R L} I^{q+r}|v(s)|(T) \\
& +\frac{1}{\Gamma(q+r)}\left|\int_{0}^{\tau_{1}}\left[\left(\tau_{2}-s\right)^{q+r-1}-\left(\tau_{1}-s\right)^{q+r-1}\right] v(s) d s+\int_{\tau_{1}}^{\tau_{2}}\left(\tau_{2}-s\right)^{q+r-1} v(s) d s\right| \\
\leq & \|p\|\left\{\frac{1}{|\Omega|}\left(\left|\Omega_{2}\right| \frac{\Gamma(q)}{\Gamma(q+r)}\left|\tau_{1}^{q+r-1}-\tau_{2}{ }^{q+r-1}\right|\right) \frac{T^{q+r}}{\Gamma(q+r+1)}\right. \\
& \left.+\frac{1}{\Gamma(q+r+1)}\left[\left|\tau_{2}{ }^{q+r}-\tau_{1}^{q+r}\right|+2\left(\tau_{2}-\tau_{1}\right)^{q+r}\right]\right\} .
\end{aligned}
$$

Obviously the right hand of the above inequality tends to zero as $\tau_{2} \rightarrow \tau_{1}$ independent of $x \in B_{r}$. So $\left\{\mathcal{L}\left(v_{n}\right)\right\}$ is an equi-continuous sequence. In consequence, the Arzelá-Ascoli theorem applies and hence there exists a uniformly convergent subsequence $\left\{v_{n}\right\}$ (labeled as $\left\{v_{n}\right\}$ again) such that $\mathcal{L}\left(v_{n}\right) \rightarrow \mathcal{L}(v)$. So $\mathcal{L}(v) \in \mathcal{L}\left(S_{F, x}\right)$ and hence $\mathcal{B}(x)=\mathcal{L}\left(S_{F, x}\right)$ is compact for all $x \in B_{r}$. Therefore, $\mathcal{B}(x)$ is compact.

In order to show that $\mathcal{B}(x)$ is convex for all $x \in \mathcal{Z}$, let $z_{1}, z_{2} \in \mathcal{B}(x)$. We select $v_{1}, v_{2} \in S_{F, x}$ such that

$$
z_{i}(t)=\frac{1}{\Omega}\left(\Omega_{1}-\Omega_{2} \frac{\Gamma(q)}{\Gamma(q+r)} t^{q+r-1}\right) R L I^{q+r} v_{i}(T)+{ }^{R L} I^{q+r} v_{i}(t), i=1,2,
$$


for almost all $t \in[0, T]$. Then, for $0 \leq \lambda \leq 1$, we have

$$
\begin{aligned}
& {\left[\lambda z_{1}+(1-\lambda) z_{2}\right](t)={ }^{R L} I^{q+r}\left[\lambda v_{1}(s)+(1-\lambda) v_{2}(s)\right](t)} \\
& +\frac{1}{\Omega}\left(\Omega_{1}-\Omega_{2} \frac{\Gamma(q)}{\Gamma(q+r)} t^{q+r-1}\right) R L I^{q+r}\left[\lambda v_{1}(s)+(1-\lambda) v_{2}(s)\right](T)
\end{aligned}
$$

So $S_{F, u}$ is convex as $\lambda v_{1}(s)+(1-\lambda) v_{2}(s) \in S_{F, x}$. Thus $\lambda z_{1}+(1-\lambda) z_{2} \in \mathcal{B}(x)$. Therefore, $\mathcal{B}$ is convex-valued. The proof for $\mathcal{A}$ is similar.

We split the remaining proof in several steps and claims.

Step 1: $\mathcal{A}$ is a multi-valued contraction on $\mathcal{Z}$. Take $x, y \in \mathcal{Z}$ and $h_{1} \in \mathcal{A} x$. Then, for each $t \in[0, T]$, there exists $v_{1}(t) \in F(t, x(t))$ such that

$$
\begin{aligned}
h_{1}(t)= & \frac{1}{\Omega}\left(\left(\Omega_{2} \frac{\Gamma(q)}{\Gamma(q+r)} t^{q+r-1}-\Omega_{1}\right) \sum_{j=1}^{n} \sigma_{j} \rho_{j} I_{\eta_{j}, \kappa_{j}}^{\alpha_{j}, \beta_{j} R L} I^{q+r} v_{1}\left(\delta_{j}\right)\right. \\
& \left.+\left(\Omega_{3}-\Omega_{4} \frac{\Gamma(q)}{\Gamma(q+r)} t^{q+r-1}\right) \sum_{i=1}^{m} \gamma_{i} \bar{\rho}_{i} I_{\bar{\eta}_{j}, \bar{\kappa}_{i}}^{\bar{\alpha}_{i}, \bar{\beta}_{i} R L} I^{q+r} v_{1}\left(\xi_{i}\right)\right) .
\end{aligned}
$$

Since $H_{d}(F(t, x), F(t, y)) \leq\|k\|\|x-y\|$, therefore, we can find $\hat{w} \in F(t, y)$ satisfying

$$
\left|v_{1}(t)-\hat{w}\right| \leq\|k\|\|x-y\| .
$$

Define $K(t)=\left\{\hat{w} \in \mathbb{R}:\left|v_{1}(t)-\hat{w}\right| \leq\|k\|\|x-y\|\right\}$. Then the multivalued operator $U$ defined by $U(t)=S_{F, y} \cap K(t)$, is measurable and nonempty. Let $v_{2}$ be a measurable selection for $U$, which exists by Kuratowski-Ryll-Nardzewski's selection theorem [27]. Then $v_{2}(t) \in F(t, y(t))$ and for each $t \in[0, T]$, we have $\left|v_{1}(t)-v_{2}(t)\right| \leq\|k\|\|x-y\|$ a.e. on $[0, T]$.

For each $t \in[0, T]$, let us define

$$
\begin{aligned}
h_{2}(t)= & \frac{1}{\Omega}\left(\left(\Omega_{2} \frac{\Gamma(q)}{\Gamma(q+r)} t^{q+r-1}-\Omega_{1}\right) \sum_{j=1}^{n} \sigma_{j} \rho_{j} I_{\eta_{j}, \kappa_{j}}^{\alpha_{j}, \beta_{j} R L} I^{q+r} v_{2}\left(\delta_{j}\right)\right. \\
& \left.+\left(\Omega_{3}-\Omega_{4} \frac{\Gamma(q)}{\Gamma(q+r)} t^{q+r-1}\right) \sum_{i=1}^{m} \gamma_{i}{ }^{\overline{\rho_{i}}} I_{\bar{\eta}_{j}, \bar{\kappa}_{i}}^{\bar{\alpha}_{i}, \bar{\beta}_{i} R L} I^{q+r} v_{2}\left(\xi_{i}\right)\right) .
\end{aligned}
$$

It follows that $h_{2} \in \mathcal{A} y$ and 


$$
\begin{aligned}
&\left|h_{1}(t)-h_{2}(t)\right| \\
& \leq \frac{1}{|\Omega|}\left(\left[\left|\Omega_{2}\right| \frac{\Gamma(q)}{\Gamma(q+r)} T^{q+r-1}+\left|\Omega_{1}\right|\right] \sum_{j=1}^{n}\left|\sigma_{j}\right| \rho_{j} I_{\eta_{j}, \kappa_{j}}^{\alpha_{j}, \beta_{j} R L} I^{q+r}\left(\left|v_{1}-v_{2}\right|\right)\left(\delta_{j}\right)\right. \\
&\left.+\left[\left|\Omega_{3}\right|+\left|\Omega_{4}\right| \frac{\Gamma(q)}{\Gamma(q+r)} T^{q+r-1}\right] \sum_{i=1}^{m}\left|\gamma_{i}\right| \bar{\rho}_{i} \bar{\eta}_{\overline{\eta_{i}}, \bar{\kappa}_{i}}^{\bar{\alpha}_{i} \bar{\beta}_{i} R L} I^{q+r}\left(\left|v_{1}-v_{2}\right|\right)\left(\xi_{i}\right)\right) \\
& \leq\|k\|\|x-y\| \frac{1}{|\Omega|}\left\{\left[\left|\Omega_{2}\right| \frac{\Gamma(q)}{\Gamma(q+r)} T^{q+r-1}+\left|\Omega_{1}\right|\right] \times\right. \\
& \times \sum_{j=1}^{n}\left|\sigma_{j}\right| \rho_{j}^{\left(-\beta_{j}\right)} \delta_{j}^{\rho_{j}\left(\alpha_{j}+\eta_{j}\right)+\kappa_{j}+q+r} \frac{\Gamma\left(\frac{\rho_{j} \eta_{j}+\rho_{j}+q+r}{\rho_{j}}\right)}{\Gamma\left(\frac{\rho_{j} \eta_{j}+\rho_{j} \alpha_{j}+\rho_{j}+q+r}{\rho_{j}}\right)} \\
&+\left[\left|\Omega_{3}\right|+\left|\Omega_{4}\right| \frac{\Gamma(q)}{\Gamma(q+r)} T^{q+r-1}\right] \times \\
& \times \sum_{i=1}^{m}\left|\gamma_{i}\right| \mid \bar{\rho}_{i}\left(-\bar{\beta}_{i}\right) \\
&=\|k\| \Lambda\|x-y\| . \\
&\left.\bar{\xi}_{i}^{\bar{\rho}_{i}\left(\bar{\alpha}_{i}+\bar{\eta}_{i}\right)+\bar{\kappa}_{i}+q+r} \frac{\Gamma\left(\frac{\bar{\rho}_{i} \bar{\eta}_{i}+\bar{\rho}_{i}+q+r}{\bar{\rho}_{i}}\right)}{\Gamma\left(\frac{\bar{\rho}_{i} \bar{\eta}_{i}+\bar{\rho}_{i} \bar{\alpha}_{i}+\bar{\rho}_{i}+q+r}{\bar{\rho}_{i}}\right)}\right\}
\end{aligned}
$$

Consequently,

$$
\left\|h_{1}-h_{2}\right\| \leq\|k\| \Lambda\|x-y\| .
$$

Interchanging the roles of $x$ and $y$, we obtain an analogous inequality:

$$
H_{d}(\mathcal{A} x, \mathcal{A} y) \leq\|k\| \Lambda\|x-y\|,
$$

which, together with the condition $\|k\| \Lambda<1$, implies that $\mathcal{A}$ is a multivalued contraction.

Step 2: We show that $\mathcal{B}$ is compact and upper semicontinuous through certain claims.

CLAIM I: $\mathcal{B}$ maps bounded sets into bounded sets in $\mathcal{Z}$.

Let $B_{\rho}=\{x \in \mathcal{Z}:\|x\| \leq \rho\}$ be a bounded ball in $\mathcal{Z}$ with $\rho>0$. Then, for each $h \in \mathcal{G}(x), x \in$ $B_{\rho}$, we can find $v \in S_{F, x}$ satisfying

$$
h(t)=\frac{1}{\Omega}\left(\Omega_{1}-\Omega_{2} \frac{\Gamma(q)}{\Gamma(q+r)} t^{q+r-1}\right) R{ }^{R L} I^{q+r} v(T)+{ }^{R L} I^{q+r} v(t) .
$$

Then we have

$$
\begin{aligned}
|h(x)| & \leq \frac{1}{|\Omega|}\left(\left|\Omega_{1}\right|+\left|\Omega_{2}\right| \frac{\Gamma(q)}{\Gamma(q+r)} t^{q+r-1}\right){ }^{R L} I^{q+r}|v(T)|+{ }^{R L} I^{q+r}|v(t)| \\
& \leq\|p\|\left\{\frac{1}{\Gamma(q+r+1)|\Omega|}\left(\left|\Omega_{1}\right| T^{q+r}+\left|\Omega_{2}\right| \frac{\Gamma(q)}{\Gamma(q+r)} T^{2 q+2 r-1}\right)+\frac{T^{q+r}}{\Gamma(q+r+1)}\right\},
\end{aligned}
$$

and consequently,

$$
\|h\| \leq\|p\|\left\{\frac{1}{\Gamma(q+r+1)|\Omega|}\left(\left|\Omega_{1}\right| T^{q+r}+\left|\Omega_{2}\right| \frac{\Gamma(q)}{\Gamma(q+r)} T^{2 q+2 r-1}\right)+\frac{T^{q+r}}{\Gamma(q+r+1)}\right\} .
$$

CLAIM II: $\mathcal{B}$ maps bounded sets into equi-continuous sets. 
Let $\tau_{1}, \tau_{2} \in[0, T]$ with $\tau_{1}<\tau_{2}$ and $x \in B_{\rho}$. For each $h \in \mathcal{G}(x)$, we obtain

$$
\begin{aligned}
& \left|h\left(\tau_{2}\right)-h\left(\tau_{1}\right)\right| \\
\leq & \frac{1}{|\Omega|}\left(\left|\Omega_{2}\right| \frac{\Gamma(q)}{\Gamma(q+r)}\left|\tau_{1}^{q+r-1}-\tau_{2}^{q+r-1}\right|\right){ }^{R L} I^{q+r}|v(s)|(T) \\
& +\frac{1}{\Gamma(q+r)}\left|\int_{0}^{\tau_{1}}\left[\left(\tau_{2}-s\right)^{q+r-1}-\left(\tau_{1}-s\right)^{q+r-1}\right] v(s) d s+\int_{\tau_{1}}^{\tau_{2}}\left(\tau_{2}-s\right)^{q+r-1} v(s) d s\right| \\
\leq & \|p\|\left\{\frac{1}{|\Omega|}\left(\left|\Omega_{2}\right| \frac{\Gamma(q)}{\Gamma(q+r)}\left|\tau_{1}^{q+r-1}-\tau_{2}^{q+r-1}\right|\right) \frac{T^{q+r}}{\Gamma(q+r+1)}\right. \\
& \left.+\frac{1}{\Gamma(q+r+1)}\left[\left|\tau_{2}^{q+r}-\tau_{1}^{q+r}\right|+2\left(\tau_{2}-\tau_{1}\right)^{q+r}\right]\right\} \rightarrow 0 \text { as } \tau_{2}-\tau_{1} \rightarrow 0
\end{aligned}
$$

independently of $x \in B_{r}$. Then, by the Arzelá-Ascoli theorem, we deduce that $\mathcal{B}: \mathcal{Z} \rightarrow \mathcal{P}(\mathcal{Z})$ is completely continuous.

Thus it follows by Claims I and II that $\mathcal{B}$ is completely continuous. Hence, by Proposition 1.2 in [28], it will be upper semicontinuous once it is shown to be closed graph. This will be shown in the next claim.

CLAIM III: $\mathcal{B}$ has a closed graph.

Letting $x_{n} \rightarrow x_{*}, h_{n} \in \mathcal{B}\left(x_{n}\right)$ and $h_{n} \rightarrow h_{*}$, we show that $h_{*} \in \mathcal{B}\left(x_{*}\right)$. For $h_{n} \in \mathcal{B}\left(x_{n}\right)$, we can find $v_{n} \in S_{F, x_{n}}$ such that, for each $t \in[0, T]$,

$$
h_{n}(t)=\frac{1}{\Omega}\left(\Omega_{1}-\Omega_{2} \frac{\Gamma(q)}{\Gamma(q+r)} t^{q+r-1}\right) R L I^{q+r} v_{n}(T)+{ }^{R L} I^{q+r} v_{n}(t) .
$$

We will show that there exists $v_{*} \in S_{F, x_{*}}$ such that for each $t \in[0, T]$,

$$
h_{*}(t)=\frac{1}{\Omega}\left(\Omega_{1}-\Omega_{2} \frac{\Gamma(q)}{\Gamma(q+r)} t^{q+r-1}\right) R L I^{q+r} v_{*}(T)+{ }^{R L} I^{q+r} v_{*}(t) .
$$

If we consider the linear operator $\Theta: L^{1}([0, T], \mathbb{R}) \rightarrow \mathcal{Z}$ given by

$$
v \mapsto \Theta(v)(t)=\frac{1}{\Omega}\left(\Omega_{1}-\Omega_{2} \frac{\Gamma(q)}{\Gamma(q+r)} t^{q+r-1}\right) R L I^{q+r} v(T)+{ }^{R L} I^{q+r} v(t),
$$

then we note that

$$
\begin{aligned}
\left\|h_{n}-h_{*}\right\|= & \|{ }^{R L} I^{q+r}\left(v_{n}(s)-v_{*}(s)\right)(t) \\
& +\frac{1}{\Omega}\left(\Omega_{1}-\Omega_{2} \frac{\Gamma(q)}{\Gamma(q+r)} t^{q+r-1}\right) R L I^{q+r}\left(v_{n}(s)-v_{*}(s)\right)(T) \| \rightarrow 0,
\end{aligned}
$$

as $n \rightarrow \infty$.

Thus, it follows by a closed graph result [29] that $\Theta \circ S_{F}$ is a closed graph operator. Further, let $h_{n}(t) \in \Theta\left(S_{F, x_{n}}\right)$. Since $x_{n} \rightarrow x_{*}$, we have that

$$
h_{*}(t)=\frac{1}{\Omega}\left(\Omega_{1}-\Omega_{2} \frac{\Gamma(q)}{\Gamma(q+r)} t^{q+r-1}\right) R L I^{q+r} v_{*}(T)+{ }^{R L} I^{q+r} v_{*}(t),
$$

for some $v_{*} \in S_{F, x_{*}}$. Hence $\mathcal{B}$ has a closed graph. Thus, the operator $\mathcal{B}$ is compact and upper semicontinuous. 
Step 3: Now, we establish that $\mathcal{A}(x)+\mathcal{B}(x) \subset B_{r}$ for all $x \in B_{r}$. Take arbitrary elements $x \in B_{r}$ with $r>\|p\| \Phi\left(\Phi\right.$ defined by (6)) and $h \in \mathcal{B}$. Then, selecting $v \in S_{F, x}$, we have

$$
\begin{aligned}
& h(t)=\frac{1}{\Omega}\left(\left(\Omega_{1}-\Omega_{2} \frac{\Gamma(q)}{\Gamma(q+r)} t^{q+r-1}\right) R L I^{q+r} v(T)\right. \\
& +\left(\Omega_{2} \frac{\Gamma(q)}{\Gamma(q+r)} t^{q+r-1}-\Omega_{1}\right) \sum_{j=1}^{n} \sigma_{j}^{\rho_{j}} I_{\eta_{j}, \kappa_{j}}^{\alpha_{j}, \beta_{j} R L} I^{q+r} v\left(\delta_{j}\right) \\
& \left.+\left(\Omega_{3}-\Omega_{4} \frac{\Gamma(q)}{\Gamma(q+r)} t^{q+r-1}\right) \sum_{i=1}^{m} \gamma_{i}{ }^{\overline{\rho_{i}}} I_{\eta_{j}, \bar{\kappa}_{i}}^{\bar{\alpha}_{i} \overline{\bar{i}}_{i} R L} I^{q+r} v\left(\xi_{i}\right)\right)+{ }^{R L} I^{q+r} v(t), t \in[0, T] .
\end{aligned}
$$

Then we have

$$
\begin{aligned}
&|h(x)| \leq\|p\|\left\{\frac { 1 } { \Gamma ( q + r + 1 ) | \Omega | } \left(\left|\Omega_{1}\right| T^{q+r}+\left|\Omega_{2}\right| \frac{\Gamma(q)}{\Gamma(q+r)} T^{2 q+2 r-1}\right.\right. \\
&+\left[\left|\Omega_{2}\right| \frac{\Gamma(q)}{\Gamma(q+r)} T^{q+r-1}+\left|\Omega_{1}\right|\right] \times \\
& \times \sum_{j=1}^{n}\left|\sigma_{j}\right| \rho_{j}^{\left(-\beta_{j}\right)} \delta_{j}^{\rho_{j}\left(\alpha_{j}+\eta_{j}\right)+\kappa_{j}+q+r} \frac{\Gamma\left(\frac{\rho_{j} \eta_{j}+\rho_{j}+q+r}{\rho_{j}}\right)}{\Gamma\left(\frac{\rho_{j} \eta_{j}+\rho_{j} \alpha_{j}+\rho_{j}+q+r}{\rho_{j}}\right)} \\
&+\left[\left|\Omega_{3}\right|+\left|\Omega_{4}\right| \frac{\Gamma(q)}{\Gamma(q+r)} T^{q+r-1}\right] \times \\
& \times \sum_{i=1}^{m}\left|\gamma_{i}\right| \mid \bar{\rho}_{i}\left(-\bar{\beta}_{i}\right) \\
&\left.\left.\xi_{i}^{\bar{\rho}_{i}\left(\bar{\alpha}_{i}+\bar{\eta}_{i}\right)+\bar{\kappa}_{i}+q+r} \frac{\Gamma\left(\frac{\bar{\rho}_{i} \bar{\eta}_{i}+\bar{\rho}_{i}+q+r}{\bar{\rho}_{i}}\right)}{\Gamma\left(\frac{\overline{\rho_{i}} \bar{\eta}_{i}+\bar{\rho}_{i} \bar{\alpha}_{i}+\bar{\rho}_{i}+q+r}{\bar{\rho}_{i}}\right)}\right)+\frac{T^{q+r}}{\Gamma(q+r+1)}\right\},
\end{aligned}
$$

which leads to

$$
\|h\| \leq\|p\| \Phi<r
$$

This shows that $\mathcal{A}(x)+\mathcal{B}(x) \subset B_{r}$ for all $x \in B_{r}$.

Thus, the operators $\mathcal{A}$ and $\mathcal{B}$ verify the hypothesis of Krasnosel'skii's multivalued fixed point theorem and hence there exists a solution $x \in \mathcal{A}(x)+\mathcal{B}(x)$ in $B_{r}$. Therefore there exists a solution of the problem (1) and (2) in $B_{r}$ which completes the proof.

Example 2. Consider problem (8) with the multi-valued map $F:[0,2] \times \mathbb{R} \rightarrow \mathcal{P}(\mathbb{R})$ defined by

$$
x \rightarrow F(t, x)=\left[\frac{\sin ^{2} t}{t^{2}+1}, \frac{1}{5\left(t^{2}+2 t+1\right)} \tan ^{-1} x+\frac{\sin ^{2} t}{t^{2}+1}\right] .
$$

Observe that

$$
\|F(t, x)\|_{\mathcal{P}}:=\sup \{|y|: y \in F(t, x)\} \leq \frac{\pi}{10\left(t^{2}+2 t+1\right)}+\frac{\sin ^{2} t}{t^{2}+1}:=p(t),
$$

and $H_{d}(F(t, x), F(t, y)) \leq(1 / 5)|x-y|$, for $x, y \in \mathbb{R}$. By using the data in problem (8), we find that $\Lambda \approx 4.209846716$. Setting $\|k\|=1 / 5$, we find that $\|k\| \Lambda \approx 0.8419693432<1$. Clearly all the assumptions of Theorem 2 hold true, and consequently, problem (8) with $F(t, x)$ given by (12) has a solution by Theorem 2 on $[0,2]$. 


\subsection{Existence Result via Wegrzyk's Fixed Point Theorem}

In this subsection we apply Wegrzyk's fixed point theorem [30] to prove an existence result for problem (1) and (2) when the right hand side of the inclusions (1) is not necessarily nonconvex valued.

Let us first recall that a multivalued operator $\mathcal{Q}: X \rightarrow \mathcal{P}_{c l}(X)$ is a generalized contraction if and only if there is a strict comparison function $\delta: \mathbb{R}_{+} \rightarrow \mathbb{R}_{+}$(continuous, strictly increasing and $\sum_{n=1}^{\infty} \delta^{n}(t)<\infty$, for each $t>0$ [31]) satisfying $H_{d}(\mathcal{Q}(x), \mathcal{Q}(y)) \leq \delta(d(x, y))$ for each $x, y \in X$. Here $\mathcal{P}_{c l}(X)=\{Y \in \mathcal{P}(X): Y$ is closed $\}$.

Lemma 3. (Wegrzyk's fixed point theorem [30]). Let $(X, d)$ be a complete metric space. If $\mathcal{Q}: X \rightarrow P_{c l}(X)$ is a generalized contraction, then Fix $\mathcal{Q} \neq \varnothing$.

Theorem 3. Assume that

$\left(A_{1}\right) F:[0, T] \times \mathbb{R} \rightarrow \mathcal{P}_{c p}(\mathbb{R})$ is such that $F(\cdot, x):[0, T] \rightarrow \mathcal{P}_{c p}(\mathbb{R})$ is measurable for each $x \in \mathbb{R}$ $\left(\mathcal{P}_{c p}(\mathbb{R})=\{W \in \mathcal{P}(\mathbb{R}): W\right.$ is compact $\left.\}\right) ;$

$\left(A_{2}\right)$ There exists $\mu \in C\left([0, T], \mathbb{R}^{+}\right)$with $d(0, F(t, 0)) \leq \mu(t)$ for almost all $t \in[0, T]$ and a strictly increasing function $v: \mathbb{R}_{+} \rightarrow \mathbb{R}_{+}$such that

$$
H_{d}(F(t, x), F(t, \bar{x})) \leq \mu(t) v(|x-\bar{x}|), x, \bar{x} \in \mathbb{R}
$$

If $\varepsilon v: \mathbb{R}_{+} \rightarrow \mathbb{R}_{+}$is a strict comparison function, where $\varepsilon=\|\mu\| \Phi(\Phi$ is defined by (6)), then the boundary value problem (1) and (2) has at least one solution on $[0, T]$.

Proof. Suppose that $\varepsilon v: \mathbb{R}_{+} \rightarrow \mathbb{R}_{+}$is a strict comparison function. Notice that $F(\cdot, x(\cdot))$ is measurable and has a measurable selection $v(\cdot)$ by the assumptions $\left(A_{1}\right)$ and $\left(A_{2}\right)$ (see Theorem III.6 [32]). As $\mu \in C([0, T], \mathbb{R})$, we have

$$
|v(t)| \leq d(0, F(t, 0))+H_{d}(F(t, 0), F(t, x(t))) \leq(1+v(\|x\|)) \mu(t) .
$$

Thus the set $S_{F, x}$ is nonempty for each $x \in \mathcal{Z}$.

Now we verify that the operator $\mathcal{G}$ satisfies the hypothesis of Lemma 3. Let us first show that $\mathcal{G}(x) \in \mathcal{P}_{c l}(\mathcal{Z})$ for each $x \in \mathcal{Z}$. Let $\left\{u_{n}\right\}_{n \geq 0} \in \mathcal{G}(x)$ be such that $u_{n} \rightarrow u$ in $\mathcal{Z}$ as $n \rightarrow \infty$. Then $u \in \mathcal{Z}$ and we can find $v_{n} \in S_{F, x_{n}}$ such that, for each $t \in[0, T]$,

$$
\begin{aligned}
u_{n}(t)= & \frac{1}{\Omega}\left(\left(\Omega_{1}-\Omega_{2} \frac{\Gamma(q)}{\Gamma(q+r)} t^{q+r-1}\right){ }^{R L} I^{q+r} v_{n}(T)\right. \\
& +\left(\Omega_{2} \frac{\Gamma(q)}{\Gamma(q+r)} t^{q+r-1}-\Omega_{1}\right) \sum_{j=1}^{n} \sigma_{j} \rho_{j} I_{\eta_{j}, \kappa_{j}}^{\alpha_{j}, \beta_{j} R L} I^{q+r} v_{n}\left(\delta_{j}\right) \\
& \left.+\left(\Omega_{3}-\Omega_{4} \frac{\Gamma(q)}{\Gamma(q+r)} t^{q+r-1}\right) \sum_{i=1}^{m} \gamma_{i}{ }^{\bar{\rho}_{i}} I_{\bar{\eta}_{j}, \bar{\kappa}_{i}}^{\bar{\alpha}_{i}, \bar{\beta}_{i} R L} I^{q+r} v_{n}\left(\xi_{i}\right)\right)+{ }^{R L} I^{q+r} v_{n}(t) .
\end{aligned}
$$

Since $F$ is compact valued, we pass onto a subsequence (if necessary) to get that $v_{n}$ converges to $v$ in $L^{1}([0, T], \mathbb{R})$. So $v \in S_{F, x}$ and for each $t \in[0, T]$, we have 


$$
\begin{aligned}
u_{n}(t) \rightarrow v(t)= & \frac{1}{\Omega}\left(\left(\Omega_{1}-\Omega_{2} \frac{\Gamma(q)}{\Gamma(q+r)} t^{q+r-1}\right) R L I^{q+r} v(T)\right. \\
& +\left(\Omega_{2} \frac{\Gamma(q)}{\Gamma(q+r)} t^{q+r-1}-\Omega_{1}\right) \sum_{j=1}^{n} \sigma_{j} \rho_{j} I_{\eta_{j}, \kappa_{j}}^{\alpha_{j}, \beta_{j} R L} I^{q+r} v\left(\delta_{j}\right) \\
& \left.+\left(\Omega_{3}-\Omega_{4} \frac{\Gamma(q)}{\Gamma(q+r)} t^{q+r-1}\right) \sum_{i=1}^{m} \gamma_{i}^{\bar{\rho}_{i}} I_{\bar{\eta}_{j}, \bar{\alpha}_{i}}^{\bar{\alpha}_{i}, \bar{\beta}_{j} R L} I^{q+r} v\left(\xi_{i}\right)\right)+{ }^{R L} I^{q+r} v(t) .
\end{aligned}
$$

Therefore, $u \in \mathcal{G}(x)$.

Next we establish that

$$
H_{d}(\mathcal{G}(x), \mathcal{G}(\bar{x})) \leq \varepsilon v(\|x-\bar{x}\|) \text { for each } x, \bar{x} \in \mathcal{Z} .
$$

Let $x, \bar{x} \in \mathcal{Z}$ and $h_{1} \in \mathcal{G}(x)$. Then there exists $v_{1}(t) \in F(t, x(t))$ such that, for each $t \in[0, T]$,

$$
\begin{aligned}
h_{1}(t)= & \frac{1}{\Omega}\left(\left(\Omega_{1}-\Omega_{2} \frac{\Gamma(q)}{\Gamma(q+r)} t^{q+r-1}\right) R L I^{q+r} v_{1}(T)\right. \\
& +\left(\Omega_{2} \frac{\Gamma(q)}{\Gamma(q+r)} t^{q+r-1}-\Omega_{1}\right) \sum_{j=1}^{n} \sigma_{j} \rho_{j} I_{\eta_{j}, \kappa_{j}}^{\alpha_{j}, \beta_{j} R L} I^{q+r} v_{1}\left(\delta_{j}\right) \\
& \left.+\left(\Omega_{3}-\Omega_{4} \frac{\Gamma(q)}{\Gamma(q+r)} t^{q+r-1}\right) \sum_{i=1}^{m} \gamma_{i}{ }^{\bar{\rho}_{i}} I_{\eta_{\bar{\eta}}, \bar{\alpha}_{i}, \bar{\beta}_{i} R L}^{\bar{\alpha}_{i}} I^{q+r} v_{1}\left(\xi_{i}\right)\right)+{ }^{R L} I^{q+r} v_{1}(t)
\end{aligned}
$$

By $\left(A_{2}\right)$, we have

$$
H_{d}(F(t, x), F(t, \bar{x})) \leq \mu(t) v(|x(t)-\bar{x}(t)|) .
$$

In consequence, we can find $w \in F(t, \bar{x}(t))$ satisfying

$$
\left|v_{1}(t)-w(t)\right| \leq \mu(t) v(|x(t)-\bar{x}(t)|), t \in[0, T] .
$$

Introduce $\mathcal{W}:[0, T] \rightarrow \mathcal{P}(\mathbb{R})$ by

$$
\mathcal{W}(t)=\left\{w \in \mathbb{R}:\left|v_{1}(t)-w\right| \leq \mu(t) v(|x(t)-\bar{x}(t)|)\right\} .
$$

By Proposition III.4 [32], the multivalued operator $\mathcal{W}(t) \cap F(t, \bar{x}(t))$ is measurable and there exists a function $v_{2}(t)$ which is a measurable selection for $\mathcal{W}$. Hence, $v_{2}(t) \in F(t, \bar{x}(t))$ and we have $\left|v_{1}(t)-v_{2}(t)\right| \leq \mu(t) v(|x(t)-\bar{x}(t)|)$, for each $t \in[0, T]$.

For each $t \in[0, T]$, let us define

$$
\begin{aligned}
h_{2}(t)= & \frac{1}{\Omega}\left(\left(\Omega_{1}-\Omega_{2} \frac{\Gamma(q)}{\Gamma(q+r)} t^{q+r-1}\right) R L\right. \\
& +\left(\Omega_{2} \frac{\Gamma(q)}{\Gamma(q+r)} t^{q+r} t^{q+r-1}-\Omega_{1}\right) \sum_{j=1}^{n} \sigma_{j} \rho^{\rho} I_{\eta_{j}, \kappa_{j}}{ }^{\alpha_{j}, \beta_{j} R L} I^{q+r} v_{2}\left(\delta_{j}\right) \\
& \left.+\left(\Omega_{3}-\Omega_{4} \frac{\Gamma(q)}{\Gamma(q+r)} t^{q+r-1}\right) \sum_{i=1}^{m} \gamma_{i}{ }^{\overline{\rho_{i}}} I_{\bar{\eta}_{j}, \bar{\kappa}_{i}}^{\bar{\alpha}_{i}, \bar{\beta}_{i} R L} I^{q+r} v_{2}\left(\xi_{i}\right)\right)+{ }^{R L} I^{q+r} v_{2}(t) .
\end{aligned}
$$

Thus, 


$$
\begin{aligned}
& \left|h_{1}(t)-h_{2}(t)\right| \\
& \leq \frac{1}{|\Omega|}\left(\left[\left|\Omega_{1}\right|+\left|\Omega_{2}\right| \frac{\Gamma(q)}{\Gamma(q+r)} T^{q+r-1}\right] R L I^{q+r}\left(\left|v_{1}-v_{2}\right|\right)(T)\right. \\
& +\left[\left|\Omega_{2}\right| \frac{\Gamma(q)}{\Gamma(q+r)} T^{q+r-1}+\left|\Omega_{1}\right|\right] \sum_{j=1}^{n}\left|\sigma_{j}\right| \rho_{j} I_{\eta_{j}, \kappa_{j}}^{\alpha_{j}, \beta_{j} R L} I^{q+r}\left(\left|v_{1}-v_{2}\right|\right)\left(\delta_{j}\right) \\
& \left.+\left[\left|\Omega_{3}\right|+\left|\Omega_{4}\right| \frac{\Gamma(q)}{\Gamma(q+r)} T^{q+r-1}\right] \sum_{i=1}^{m}\left|\gamma_{i}\right|{ }^{\bar{\rho}_{i}} I_{\bar{\eta}_{i}, \bar{\kappa}_{i}, \bar{\kappa}_{i} R L} I^{q+r}\left(\left|v_{1}-v_{2}\right|\right)\left(\xi_{i}\right)\right) \\
& +{ }^{R L} I^{q+r}\left(\left|v_{1}-v_{2}\right|\right)(t) \\
& \leq\|\mu\| v(\|x-\bar{x}\|)\left\{\frac { 1 } { \Gamma ( q + r + 1 ) | \Omega | } \left(\left|\Omega_{1}\right| T^{q+r}+\left|\Omega_{2}\right| \frac{\Gamma(q)}{\Gamma(q+r)} T^{2 q+2 r-1}\right.\right. \\
& +\left[\left|\Omega_{2}\right| \frac{\Gamma(q)}{\Gamma(q+r)} T^{q+r-1}+\left|\Omega_{1}\right|\right] \times \\
& \times \sum_{j=1}^{n}\left|\sigma_{j}\right| \rho_{j}^{\left(-\beta_{j}\right)} \delta_{j}^{\rho_{j}\left(\alpha_{j}+\eta_{j}\right)+\kappa_{j}+q+r} \frac{\Gamma\left(\frac{\rho_{j} \eta_{j}+\rho_{j}+q+r}{\rho_{j}}\right)}{\Gamma\left(\frac{\rho_{j} \eta_{j}+\rho_{j} \alpha_{j}+\rho_{j}+q+r}{\rho_{j}}\right)} \\
& +\left[\left|\Omega_{3}\right|+\left|\Omega_{4}\right| \frac{\Gamma(q)}{\Gamma(q+r)} T^{q+r-1}\right] \times \\
& \left.\left.\times \sum_{i=1}^{m}\left|\gamma_{i}\right| \mid \bar{\rho}_{i}\left(-\bar{\beta}_{i}\right) \xi_{i}^{\bar{\rho}_{i}\left(\bar{\alpha}_{i}+\bar{\eta}_{i}\right)+\bar{\kappa}_{i}+q+r} \frac{\Gamma\left(\frac{\bar{\rho}_{i} \bar{\eta}_{i}+\bar{\rho}_{i}+q+r}{\bar{\rho}_{i}}\right)}{\Gamma\left(\frac{\bar{\rho}_{i} \bar{\eta}_{i}+\bar{\rho}_{i} \bar{\alpha}_{i}+\bar{\rho}_{i}+q+r}{\bar{\rho}_{i}}\right)}\right)+\frac{T^{q+r}}{\Gamma(q+r+1)}\right\} \\
& =\|\mu\| \Phi v(\|x-\bar{x}\|) \text {. }
\end{aligned}
$$

Hence $\left\|h_{1}-h_{2}\right\| \leq \varepsilon v(\|x-\bar{x}\|)$. Analogously, one can obtain by interchanging the roles of $x$ and $\bar{x}$ that $H_{d}(\mathcal{G}(x), \mathcal{G}(\bar{x})) \leq \varepsilon v(\|x-\bar{x}\|)$ for each $x, \bar{x} \in \mathcal{Z}$. Thus $\mathcal{G}$ is a generalized contraction. So, by Lemma 3, we deduce that $\mathcal{G}$ has a fixed point $x$, which corresponds to a solution of problem (1) and (2). This finishes the proof.

Example 3. Let us consider problem (8) with $F(t, x)$ given by

$$
F(t, x)=\left[\frac{1}{6}, \frac{t+1 / 2}{1+t^{2}} \tan ^{-1} x+\frac{1}{2}\right],
$$

and note that

$$
\sup \{|x|, y \in F(t, x)\} \leq \frac{(t+1 / 2) \pi}{2\left(1+t^{2}\right)}+\frac{1}{2}, H_{d}(F(t, x), F(t, \bar{x})) \leq \frac{(t+1 / 2)}{1+t^{2}}(|x-\bar{x}|) .
$$

Fix $\mu(t)=\frac{(t+1 / 2)}{1+t^{2}}$ such that $d(0, F(t, 0)) \leq \mu(t)$ for almost all $t \in[0,2]$ and $\|\mu\| \approx 0.780330$. From Example 1, we have $\Phi \approx 4.519175132$. In consequence $\varepsilon=\|\mu\| \Phi \approx 3.526448$. Letting $v(x)=x$, all the conditions of Theorem 3 are satisfied. Therefore the problem (8) with $F(t, x)$ given by (13) has has at least one solution on $[0,2]$ by the conclusion of Theorem 3 .

\section{Conclusions}

In this paper, we presented three existence results for sequential fractional differential inclusions involving Riemmann-Liouville and Caputo type derivatives, subject to generalized fractional integral boundary conditions. These results provide different criteria for the existence of solutions for the problem at hand. The first result (Theorem 1) is obtained with the aid of the endpoint theory, while Krasnosel'skií's fixed point theorem for multivalued maps is applied to derive the second result (Theorem 2). In the third result (Theorem 3), we used Wegrzyk's fixed point theorem for generalized 
contractions to establish the existence of solutions for the given problem. It is imperative to note that Wegrzyk's fixed point theorem is a generalization of Covit and Nadler's fixed point theorem [33] in the sense that it deals with the generalized contractions. Thus Theorem 3 holds for several values of the function $v$ (defined in $\left(A_{2}\right)$ ); for example, $v(t)=\ln (1+t)^{3}, v(t)=t$ (contraction case), etc. Some interesting special cases of our results follow by fixing the parameters involved in the boundary conditions (2). For instance, our results correspond to Dirichlet boundary conditions if we take $\gamma_{i}=0$ for all $i=1,2, \ldots, m$ and $\sigma_{j}=0$ for all $j=1,2, \ldots, n$. Fixing $\gamma_{i}=0$ for all $i=1,2, \ldots, m$ and $\sigma_{j} \neq 0$ for some $j=1,2, \ldots, n$, we obtain the existence results for the boundary conditions of the form: $x(0)=0, x(T)=\sum_{j=1}^{n} \sigma_{j} \rho_{j} I_{\eta_{j}, \kappa_{j}}^{\alpha_{j}, \beta_{j}} x\left(\delta_{j}\right)$. On the other hand, we obtain the existence results for (1) associated with the boundary conditions: $x(0)=\sum_{i=1}^{m} \gamma_{i} \bar{\rho}_{i} I_{\bar{\eta}_{i}, \bar{\kappa}_{i}}^{\bar{\alpha}_{i} \bar{\beta}_{i}} x\left(\xi_{i}\right), x(T)=0$, by letting $\gamma_{i} \neq 0$ for some $i=1,2, \ldots, m$, and $\sigma_{j}=0$ for all $j=1,2, \ldots, n$. We emphasize that the existence results indicated for special forms of the boundary conditions are new.

Author Contributions: Conceptualization, J.T., S.K.N. and B.A.; formal analysis, J.T., S.K.N., B.A. and A.A.; funding acquisition, J.T.; methodology, J.T., S.K.N., B.A. and A.A. All authors have read and agreed to the published version of the manuscript.

Funding: This research received no external funding.

Acknowledgments: This research was funded by King Mongkut's University of Technology North Bangkok. Contract no. KMUTNB-61-KNOW-021.

Conflicts of Interest: The authors declare no conflict of interest.

\section{References}

1. Kisielewicz, M. Stochastic Differential Inclusions and Applications; Springer Optimization and Its Applications, 80; Springer: New York, NY, USA, 2013.

2. Podlubny, I. Fractional Differential Equations; Academic Press: New York, NY, USA, 1999.

3. Balochian, S.; Nazari, M. Stability of particular class of fractional differential inclusion systems with input delay. Control Intell. Syst. 2014, 42, 279-283. [CrossRef]

4. Wang, X.; Schiavone, P. Harmonic three-phase circular inclusions in finite elasticity. Contin. Mech. Thermodyn. 2015, 27, 739-747. [CrossRef]

5. Sun, J.; Yin, Q. Robust fault-tolerant full-order and reduced-order observer synchronization for differential inclusion chaotic systems with unknown disturbances and parameters. J. Vib. Control 2015, 21, 2134-2148. [CrossRef]

6. He, J.; Zhang, X.; Liu, L.; Wu, Y.; Cui, Y. Existence and asymptotic analysis of positive solutions for a singular fractional differential equation with nonlocal boundary conditions. Bound Value Probl. 2018. [CrossRef]

7. Zhang, X.; Liu, L.; Wu, Y. Multiple positive solutions of a singular fractional differential equation with negatively perturbed term. Math. Comput. Model. 2012, 55, 1263-1274. [CrossRef]

8. Ahmad, B.; Ntouyas, S.K. Existence results for higher order fractional differential inclusions with multi-strip fractional integral boundary conditions. Electron. J. Qual. Theory Differ. Equ. 2013, 19. [CrossRef]

9. Ntouyas, S.K.; Etemad, S.; Tariboon, J. Existence results for multi-term fractional differential inclusions. Adv. Difference Equ. 2015, 140. [CrossRef]

10. Ahmad, B.; Agarwal, R.P.; Alsaedi, A. Fractional differential equations and inclusions with semiperiodic and three-point boundary conditions. Bound. Value Probl. 2016, 28, 20. [CrossRef]

11. Ahmad, B.; Ntouyas, S.K. Existence results for fractional differential inclusions with Erdélyi-Kober fractional integral conditions. An. Ştiinţ. Univ. Ovidius Constanţa Ser. Mat. 2017, 25, 5-24. [CrossRef]

12. Cheng, Y.; Agarwal, R.P.; O’Regan, D. Existence and controllability for nonlinear fractional differential inclusions with nonlocal boundary conditions and time-varying delay. Fract. Calc. Appl. Anal. 2018, 21, 960-980. [CrossRef]

13. Abbas, S.; Benchohra, M.; Graef, J.R. Coupled systems of Hilfer fractional differential inclusions in Banach spaces. Commun. Pure Appl. Anal. 2018, 17, 2479-2493. [CrossRef] 
14. Ahmad, B.; Ntouyas, S.K.; Zhou, Y.; Alsaedi, A. A study of fractional differential equations and inclusions with nonlocal Erdélyi-Kober type integral boundary conditions. Bull. Iranian Math. Soc. 2018, 44, 1315-1328. [CrossRef]

15. Wang, J.R.; Ibrahim, A.G.; Fečkan, M.; Zhou, Y. Controllability of fractional non-instantaneous impulsive differential inclusions without compactness. IMA J. Math. Control Inform. 2019, 36, 443-460. [CrossRef]

16. Ahmad, B.; Ntouyas, S.K.; Alsaedi, A. Coupled systems of fractional differential inclusions with coupled boundary conditions. Electron. J. Differ. Equ. 2019, 2019, 21.

17. Ahmad, B.; Ntouyas, S.K.; Tariboon, J. On inclusion problems involving Caputo and Hadamard fractional derivatives. Acta Math. Univ. Comenian. 2020, 89, 169-183.

18. Benchohra, M.; Hamani, S.; Zhou, Y. Oscillation and nonoscillation for Caputo-Hadamard impulsive fractional differential inclusions. Adv. Differ. Equ. 2019, 74, 15. [CrossRef]

19. Wang, Y.; Liang, T. Mild solutions to the time fractional Navier-Stokes delay differential inclusions. Discrete Contin. Dyn. Syst. Ser. B 2019, 24, 3713-3740. [CrossRef]

20. Alsaedi, A.; Broom, A.; Ntouyas, S.K.; Ahmad, B. Nonlocal fractional boundary value problems involving mixed right and left fractional derivatives and integrals. Axioms 2020, 9, 50. [CrossRef]

21. Ntouyas, S.K.; Al-Sulami, H.H. A study of coupled systems of mixed order fractional differential equations and inclusions with coupled integral fractional boundary conditions. Adv. Differ. Equ. 2020, 73. [CrossRef]

22. Promsakon, C.; Phuangthong, N.; Ntouyas, S.K.; Tariboon, J. Nonlinear sequential Riemann-Liouville and Caputo fractional differential equations with generalized fractional integral conditions. Adv. Differ. Equ. 2018, 385. [CrossRef]

23. Kisielewicz, M. Differential Inclusions and Optimal Control; Kluwer: Dordrecht, The Netherlands, 1991.

24. Amini-Harandi, A. Endpoints of set-valued contractions in metric spaces. Nonlinear Anal. 2010, 72, 132-134. [CrossRef]

25. Ahmad, B.; Ntouyas, S.K.; Tariboon, J. A study of mixed Hadamard and Riemann-Liouville fractional integro-differential inclusions via endpoint theory. Appl. Math. Lett. 2016, 52, 9-14. [CrossRef]

26. Petrusel, A. Fixed points and selections for multivalued operators. In Seminar on Fixed Point Theory Cluj-Napoca; Babes-Bolyai University: Cluj-Napoca, Romania, 2001; Volume 2, pp. 3-22.

27. Hu, S.; Papageorgiou, N. Handbook of Multivalued Analysis, Theory I; Kluwer: Dordrecht, The Netherlands, 1997.

28. Deimling, K. Multivalued Differential Equations; Walter De Gruyter: Berlin, Germany; New York, NY, USA, 1992.

29. Lasota, A.; Opial, Z. An application of the Kakutani-Ky Fan theorem in the theory of ordinary differential equations. Bull. Acad. Polon. Sci. Ser. Sci. Math. Astronom. Phys. 1965, 13, 781-786.

30. Wegrzyk, R. Fixed Point Theorems for Multifunctions and Their Applications to Functional Equations; Instytut Matematyczny Polskiej Akademi Nauk: Warszawa, Poland, 1982. Available online: http:/ / eudml.org/doc/ 268403 (accessed on 26 June 2020).

31. Granas, A.; Dugundji, J. Fixed Point Theory; Springer: New York, NY, USA, 2003.

32. Castaing, C.; Valadier, M. Convex Analysis and Measurable Multifunctions; Lecture Notes in Mathematics 580; Springer: Berlin/Heidelberg, Germany; New York, NY, USA, 1977.

33. Covitz, H.; Nadler, S.B., Jr. Multivalued contraction mappings in generalized metric spaces. Israel J. Math. 1970, 8, 5-11. [CrossRef]

(C) 2020 by the authors. Licensee MDPI, Basel, Switzerland. This article is an open access article distributed under the terms and conditions of the Creative Commons Attribution (CC BY) license (http://creativecommons.org/licenses/by/4.0/). 